\title{
Book review: "Topological Degree Approach to Bifurcation Problems" by Michal Fečkan
}

\author{
A. Rontó
}




\title{
BOOK REVIEW: "TOPOLOGICAL DEGREE APPROACH TO BIFURCATION PROBLEMS" BY MICHAL FEČKAN
}

\author{
A. RONTÓ \\ Received 24 November, 2008
}

\begin{abstract}
Topological bifurcation theory is one of the most essential topics in mathematics. This book contains original bifurcation results for the existence of oscillations and chaotic behaviour of differential equations and discrete dynamical systems under variation of involved parameters. Using topological degree theory and a perturbation approach in dynamical systems, a broad variety of non-linear problems are studied, including: non-smooth mechanical systems with dry frictions; weakly coupled oscillators; systems with relay hysteresis; differential equations on infinite lattices of FrenkelKontorova and discretized Klein-Gordon types; blue sky catastrophes for reversible dynamical systems; buckling of beams; and discontinuous wave equations.

Precise and complete proofs, together with concrete applications with many stimulating and illustrating examples, make this book valuable to both the applied sciences and mathematical fields, ensuring the book should not only be of interest to mathematicians but to physicists and theoretically inclined engineers interested in bifurcation theory and its applications to dynamical systems and non-linear analysis.
\end{abstract}

(Publisher's description)

The theory of bifurcations is one of the most important and actively studied topics in non-linear analysis $[1,5-7,10,12-14,17]$. It has numerous applications in physics, biology, chemistry, and economics, where phenomena are often modelled by using differential or difference equations depending on parameters and it is necessary to find out which qualitative changes of the system may be caused by small changes of the parameters involved. The basic approaches here are the topological, analytical, and variational ones. When the non-linearities appearing in the equations under investigation are not smooth enough, the classical approaches relying on the differentiability fail, whereas the topological tools prove to be rather efficient. It is this wide class of bifurcation problems that is investigated in Michal Fečkan's recent monograph [11] published by Springer-Verlag in 2008. 
The book [11] is devoted to the study of various non-smooth bifurcation problems ranging from non-smooth mechanical systems [3] through ordinary differential equations on infinite non-linear lattices [21] up to undamped abstract wave equations in Hilbert spaces with applications to non-linear beam and string partial differential equations [14]. It is organised as follows.

In the introductory Chapter 1, an illustrative example is presented on the bifurcation of chaotic solutions in a differential model describing the apparatus consisting of a slender beam clamped to a rigid framework which supports two magnets. The apparatus is periodically forced using electromagnetic vibration generator. Mathematically, this leads one to the well-known periodically forced and damped Duffing equation. A Melnikov bifurcation function is obtained which determines wedge-shaped regions in the parametric space where the perturbed Duffing equation is chaotic. This example is used to outline main ideas of the approach of the book, which is based on the perturbation method from the theory of dynamical systems, the LyapunovSchmidt decomposition method [17, 20], and the Brouwer and Leray-Schauder theories of topological degree $[15,17]$.

Chapter 2 of [11] contains a brief review of some known mathematical results from functional analysis, differential topology, theory of multivalued mappings, and theory of dynamical systems, which are systematically applied in the proofs of the main results of the monograph.

The main original contribution of the book starts from Chapter 3, where bifurcations of periodic and subharmonic solutions from either periodic or homoclinic trajectories are studied for several types of differential systems, namely, for differential equations with dry frictions, weakly coupled non-linear oscillators, and forced systems with relay hysteresis. As a preparatory step to discover chaos in discontinuous systems, the existence of infinitely many subharmonics with periods tending to infinity is established for weakly discontinuous differential equations. Systems of ordinary differential equations and inclusions containing small parameters multiplying the derivatives are studied (see, e.g., [19,24]). Among the rest, classical results on the saddle-node and Poincaré-Andronov bifurcations of periodic solutions $[12,18]$ are extended to non-smooth differential equations. Many concrete examples illustrate the theory.

In Chapter 4, the existence of chaotic solutions of discontinuous differential equations is studied by extending the approach of Chapter 3. The unperturbed system is supposed to have either one homoclinic solution or a manifold filled with such solutions. Melnikov type conditions are established for proving the existence of chaos for perturbed systems. Almost-periodically and quasi-periodically forced differential inclusions are studied as well. The chapter ends with a review of recent results on the homoclinic bifurcation for other types of discontinuous differential equations.

Chapter 5 deals with the study of chaos for diffeomorphisms in the cases where the intersection of the stable and unstable manifolds of a hyperbolic fixed point is 
only topologically transversal. Topological degree methods here again prove to be an important tool. An extension of the classical Smale-Birkhoff homoclinic theorem [12] is obtained for the existence of topologically transversal homoclinic and heteroclinic points of diffeomorphisms. Bifurcations of such points are also studied. The rest of the chapter deals with the accumulation of periodic points of reversible diffeomorphisms near homoclinic points, with extensions of this phenomenon, known as the blue sky catastrophe [8-10], to chains of time-reversible oscillators.

In Chapter 6, the author studies equations on infinite non-linear lattices obtained as a result of a spatial discretisation of partial differential equations [22]. The persistence of kink travelling waves of partial differential equations under discretisation is investigated. The idea here is to consider a travelling wave equation of the lattice equation as an evolution equation in a suitable Banach space. Then the centre manifold method $[12,13,18]$ is applied to lower the dimension of this infinite dimensional evolution equation. A result on the bifurcation of periodic solutions of certain singularly perturbed ordinary differential equations is obtained, where the small parameter determining the perturbation is the discretisation step size. Statements on the bifurcation of periodic solutions are then used when analysing travelling wave solutions of the sine-Gordon and Klein-Gordon discretised lattice equations [22]. The chapter also contains a review of results on the existence of travelling waves for differential equations on two-dimensional lattices.

Chapter 7 is devoted to the study of the existence of periodic and subharmonic solutions of undamped abstract wave equations. Here, the analysis is complicated by the presence of infinitely many resonant terms, which effect is known as the problem of small divisors [16]. To overcome this difficulty, certain Diophantine-type inequalities are introduced. Then using analytical and topological arguments, the bifurcations of subharmonic solutions from homoclinic ones and the bifurcations of periodic solutions from periodic ones are investigated, with applications to several types of periodically forced non-linear beam equations. Weakly non-linear wave equations are also studied.

The final Chapter 8 concerns discontinuous wave equations with infinitely many resonances. The approach here uses that of [2], which is extended to the case of a multi-valued monotone mapping. For this purpose, the notion of a topological degree for monotone multi-valued mappings is constructed by combining the BrowderSkrypnik topological degree theory $[4,23]$ with Mawhin's coincidence index theory [17]. This topological degree is then used to extend the Krasnoselskii bifurcation theorem and theorems on bifurcations from infinity $[1,6,17]$ to multi-valued operator equations in Hilbert spaces. The resulting abstract bifurcation theorems are applied to study bifurcations of weak forced large-amplitude periodic solutions of discontinuous undamped semilinear wave equations. Finally, the author discusses the possibilities to apply the methods developed in the monograph for detecting the presence of chaos for weakly discontinuous and periodically forced semilinear wave equations. 
The book presents to the reader many sophisticated original theorems on a wide range of problems of bifurcation theory. The exposition is based on the author's recently published results that have not yet been included in any other monograph.

\section{REFERENCES}

[1] M. S. Berger, Nonlinearity and functional analysis. Lectures on nonlinear problems in mathematical analysis, ser. Pure and Applied Mathematics. New York: Academic Press [Harcourt Brace Jovanovich Publishers], 1977.

[2] J. Berkovits and V. Mustonen, "An extension of Leray-Schauder degree and applications to nonlinear wave equations," Differential Integral Equations, vol. 3, no. 5, pp. 945-963, 1990.

[3] B. Brogliato, Nonsmooth impact mechanics. Models, dynamics and control, ser. Lecture Notes in Control and Information Sciences. London: Springer-Verlag London Ltd., 1996, vol. 220.

[4] F. E. Browder, "Degree theory for nonlinear mappings," in Nonlinear functional analysis and its applications, Part 1 (Berkeley, Calif., 1983), ser. Proc. Sympos. Pure Math. Providence, RI: Amer. Math. Soc., 1986, vol. 45, pp. 203-226.

[5] R. F. Brown, A topological introduction to nonlinear analysis. Boston, MA: Birkhäuser Boston Inc., 1993.

[6] S. N. Chow and J. K. Hale, Methods of bifurcation theory, ser. Grundlehren der Mathematischen Wissenschaften [Fundamental Principles of Mathematical Science]. New York: Springer-Verlag, 1982, vol. 251.

[7] K. Deimling, Nonlinear functional analysis. Berlin: Springer-Verlag, 1985.

[8] R. L. Devaney, "Reversible diffeomorphisms and flows," Trans. Amer. Math. Soc., vol. 218, pp. 89-113, 1976.

[9] R. L. Devaney, "Blue sky catastrophes in reversible and Hamiltonian systems," Indiana Univ. Math. J., vol. 26, no. 2, pp. 247-263, 1977.

[10] R. L. Devaney, An introduction to chaotic dynamical systems. Menlo Park, CA: The Benjamin/Cummings Publishing Co. Inc., 1986.

[11] M. Fečkan, Topological degree approach to bifurcation problems., ser. Topological Fixed Point Theory and Its Applications. Dordrecht: Springer-Verlag, 2008, vol. 5.

[12] J. Guckenheimer and P. Holmes, Nonlinear oscillations, dynamical systems, and bifurcations of vector fields, ser. Applied Mathematical Sciences. New York: Springer-Verlag, 1983, vol. 42.

[13] G. Iooss and M. Adelmeyer, Topics in bifurcation theory and applications, ser. Advanced Series in Nonlinear Dynamics. River Edge, NJ: World Scientific Publishing Co. Inc., 1992, vol. 3.

[14] H. Kielhöfer, Bifurcation theory. An introduction with applications to PDEs, ser. Applied Mathematical Sciences. New York: Springer-Verlag, 2004, vol. 156.

[15] M. A. Krasnosel'skiı̌ and P. P. Zabrě̌ko, Geometrical methods of nonlinear analysis, ser. Grundlehren der Mathematischen Wissenschaften [Fundamental Principles of Mathematical Sciences]. Berlin: Springer-Verlag, 1984, vol. 263, translated from the Russian by Christian C. Fenske.

[16] S. B. Kuksin, Nearly integrable infinite-dimensional Hamiltonian systems, ser. Lecture Notes in Mathematics. Berlin: Springer-Verlag, 1993, vol. 1556.

[17] J. Mawhin, Topological degree methods in nonlinear boundary value problems, ser. CBMS Regional Conference Series in Mathematics. Providence, R.I.: American Mathematical Society, 1979, vol. 40, Expository lectures from the CBMS Regional Conference held at Harvey Mudd College, Claremont, Calif., June 9-15, 1977.

[18] M. Medved, Fundamentals of dynamical systems and bifurcation theory. Bristol: Adam Hilger Ltd., 1992. 
[19] E. F. Mishchenko, Y. S. Kolesov, A. Y. Kolesov, and N. K. Rozov, Asymptotic methods in singularly perturbed systems, ser. Monographs in Contemporary Mathematics. New York: Consultants Bureau, 1994, translated from the Russian by Irene Aleksanova.

[20] N. Rouche and J. Mawhin, Ordinary differential equations. Stability and periodic solutions, ser. Surveys and Reference Works in Mathematics. Boston, Mass.: Pitman (Advanced Publishing Program), 1980, vol. 5, translated from the French and with a preface by R. E. Gaines.

[21] A. M. Samoilenko and Y. V. Teplinskii, Countable systems of differential equations. Utrecht: VSP, 2003.

[22] A. Scott, Nonlinear science. Emergence and dynamics of coherent structures, 2nd ed., ser. Oxford Texts in Applied and Engineering Mathematics. Oxford: Oxford University Press, 2003, vol. 8.

[23] I. V. Skrypnik, Methods for analysis of nonlinear elliptic boundary value problems, ser. Translations of Mathematical Monographs. Providence, RI: American Mathematical Society, 1994, vol. 139, translated from the 1990 Russian original by Dan D. Pascali.

[24] A. N. Tihonov, "Systems of differential equations containing small parameters in the derivatives," Mat. Sbornik N. S., vol. 31(73), pp. 575-586, 1952.

Author's address

\section{A. Rontó}

Institute of Mathematics of the Academy of Sciences of the Czech Republic, branch in Brno, 22 Žižkova St., 61662 Brno, Czech Republic

E-mail address: ronto@math.cas.cz 